\title{
Quando o Ruído de Lazer Afeta a Saúde de uma Comunidade
}

\author{
Jonas Carvalho e Silva* \\ Júlia Sursis Nobre Ferro Bucher-Maluschke** \\ Christoph Käppler***
}

\begin{abstract}
Resumo
O objetivo deste manuscrito foi relatar as ações comunitárias de um grupo de moradores, pesquisadores e profissionais da saúde contra os impactos do ruído de lazer (paredões de sons automotivos) no centro histórico de Porto Nacional-TO, Brasil. Nesta localidade foi implementada, como medida compensatória, uma orla de lazer após o enchimento do lago da Usina Luis Eduardo Magalhães em 2001. O trabalho relata o contexto do surgimento desse fenômeno e a emergência das tensões sociais entre os residentes, comerciantes, gestores públicos e transeuntes que gravitam em torno da exposição ao ruído. A partir das ações de mobilização na comunidade, foi desenvolvida uma revisão junto as publicações do Escritório Europeu da Organização Mundial da Saúde que evidenciaram o ruído de lazer como um problema de saúde pública e uma das formas de poluição que mais afeta as pessoas. Dentre os impactos, destacam-se os efeitos biológicos, qualidade do sono, bem-estar e condições médicas. Apesar das evidências encontradas e das medidas de mitigação adotadas pelos gestores, o ruído ainda incomoda os moradores, o que sugere um forte fator cultural em relação a este problema. Conclui-se que as informações deste relato são promissoras para uma área ainda em desenvolvimento no Brasil e que implica a articulação interdisciplinar em torno dos efeitos dos barulhos de lazer na saúde.

Palavras-chave: ruído de lazer, saúde comunitária, hidrelétricas.
\end{abstract}

\section{When Leisure Noise Affects the Health of a Community}

\begin{abstract}
The objective of this manuscript was to report the community actions of a group of residents, researchers and health professionals against the impacts of leisure noise (car sound walls) in the historic center of Porto Nacional-TO, Brazil. In this location, as a compensatory measure, a leisure area was implemented after the filling of the Luis Eduardo Magalhães Power Plant lake in 2001. The work relates the context of the emergence of this phenomenon and the emergence of social tensions among residents, shopkeepers, public managers and passers-by who gravitate around exposure to noise. From the mobilization actions in the community, a research was developed with the publications of the European Office of the World Health Organization that highlighted leisure noise as a public health problem and one of the forms of pollution that most affects people. Among the impacts, biological effects, sleep quality, well-being and medical conditions stand out. Despite the evidence found and the mitigation measures adopted by managers, noise still disturbs residents, suggesting a strong cultural factor in relation to this problem. It is concluded that the information in this report is promising for an area still under development in Brazil and that it implies interdisciplinary articulation around the effects of leisure noise on health.
\end{abstract}

Keywords: leisure noise, community health, hydroelectric.

* Psicólogo, Mestre pelo CEULP/ULBRA e Doutor pela UNB.

** Psicóloga pela UNB, Mestre e Dra. pela Universite Catholique de Louvain. Profa. da Universidade de Fortaleza e da UNB.

*** Psicólogo, Mestre e Dr. pela Albert-Ludwigs-Universität Freiburg. Prof. Catedrático na Faculdade Technische Universität. 


\section{Introdução}

Um grupo de moradores, pesquisadores, profissionais da saúde, estudantes e militantes do movimento social do município de Porto Nacional-TO estão mobilizados em ações comunitárias de combate ao barulho, no seu centro histórico, provocado por paredões de som ${ }^{1}$. Esta cidade é impactada por uma usina hidrelétrica, e por isso teve parte da sua paisagem alterada, bem como as opções de lazer (Messias, 2012). Os paredões provocam problemas, aborrecimentos e perturbação aos moradores, especialmente os idosos, doentes crônicos, crianças, trabalhadores e estudantes (Carvalho e Silva, 2018).

Diante deste fenômeno, o grupo decidiu aprofundar o conhecimento sobre as implicações do som automotivo. Os estudos sobre os efeitos do ruído identificam-no como um problema de saúde pública e uma das formas de poluição que mais afeta as pessoas (Héroux et al., 2020). Entretanto, devido a sua natureza física, de propagar sem causar alterações permanentes, a sua avaliação pode ser complexa e subjetiva, especialmente quanto aos seus impactos sobre a saúde, muitas vezes com manifestações psicossomáticas e sociais (Bressane, Mochizuki, Caram, \& Roveda, 2016).

Apesar das garantias do direito ao sossego promulgadas em Leis nos níveis federal, estadual e municipal, observa-se que a população não tem acesso as informações sobre as complicações que a exposição ao ruído leva para a saúde (Alves, Araújo, \& Pinto, 2014; Bressane et al., 2016). Este fator se deve, por um lado, por inferências culturais, das quais associam o ruído como uma forma de lazer (Dos Santos \& Rodrigues, 2019; Hebenbrock, 2012). Por outro, o desinteresse público nesta questão e a ausência de percepção dos profissionais da saúde sobre os riscos (Héroux et al., 2020).

O grupo observou no estado da arte poucos estudos sobre os efeitos do barulho de lazer na saúde dos brasileiros. Tão pouco foram localizados documentos oficiais no Brasil que orientem a população e os pesquisadores sobre os efeitos na saúde, protocolos de pesquisas, ou de avaliações, internas ou no âmbito da cooperação internacional. A exemplo do Escritório Regional Europeu da Organização Mundial (OMS/Europa), que desenvolve programas sobre o ruído nas áreas de ambiente de vida e saúde.

Diante desta limitação, este relato tem por objetivo apresentar o contexto das reivindicações dos moradores contra o impacto do barulho provocado pelos paredões. A experiência ocorreu durante a execução do

Considera-se paredão de som qualquer aparato de som automotivo rebocado, instalado ou acoplado nos porta-malas do veículo. projeto de pesquisa "Comunidades ribeirinhas impactadas por barragens", no período de 2014 a 2018 (CAAE: 70302016.8.0000.5540). Será apresentada uma explanação sobre o surgimento do problema, as repercussões das estratégias de enfrentamento do grupo, e os resultados de uma revisão sobre os efeitos do ruído na saúde humana.

\section{Contexto do problema da comunidade com o barulho dos paredões}

Porto Nacional é uma cidade histórica localizada no Estado do Tocantins, região norte do Brasil, situada à $64 \mathrm{~km}$ da capital Palmas, com uma população de 50 mil habitantes (Brasil, 2018). Desde o início da sua povoação, no século final do século XVIII, ela mantém uma estreita relação econômica e afetiva com o rio Tocantins, que se ergueu a margem dele (Messias, 2012; Oliveira, 1997). O rio tornou-se, portanto, um patrimônio cultural da população, no qual sempre foi um espaço de lazer, mesmo que no decorrer do tempo ele tenha sido utilizado de diferentes maneiras (Carvalho e Silva \& Ertzogue, 2015).

No ano de 2001 a relação dos moradores com o rio Tocantins passou por uma nova transformação decorrente do barreamento do lago da Usina Hidrelétrica Luis Eduardo Magalhães no município de Lajeado, localizado a $117 \mathrm{~km}$. Porto Nacional foi a cidade mais atingida pelo enchimento do rio, tendo sido inundada uma parte do seu patrimônio histórico e turístico (CELTINS, 1996). O processo de deslocamento das famílias e de demolição de parte da cidade foi permeado por reivindicações da classe artística, acadêmica, dos moradores e dos movimentos sociais, que influenciaram nas compensações previstas (Foschiera, 2009; Zitzke, 2007).

Vários pesquisadores relatam os impactos negativos da construção da usina hidrelétrica na vida dos moradores do município (Carvalho e Silva \& Ertzogue, 2015; Foschiera, 2009; Jesus \& Ertzogue, 2018; Messias, 2012; Parente, 2015; Zitzke, 2007). Além dos impactos imediatos ao momento da implementação do empreendimento, nota-se os efeitos a longo prazo, provocados pelas novas dinâmicas individuais e contextuais impostas a comunidade. Carvalho e Silva (2018), identificou que após quase duas décadas da inundação, e com a extensão do agronegócio, as famílias de Porto Nacional ainda sofrem com o aumento do consumo de drogas, desemprego, violência armada e de gênero, transtornos mentais, desnutrição e perturbação do sossego.

Dentre os planos de mitigação dos impactos foi implementada uma orla nas margens do lago, localizada no centro histórico, com a proposta de ser um espaço 
de lazer e turístico da cidade (CELTINS, 1996). Nela foi construída uma ampla avenida com anfiteatro, quadras esportivas, academias, bares e restaurantes, onde ocorrem as festas públicas. O surgimento dos paredões neste local perturbam os moradores devido o alto volume e o aumento da sensação de insegurança, impedindo que grupos especiais como crianças, idosos e doentes crônicos frequentem o espaço (Carvalho e Silva, 2018; Messias, 2012).

\section{Enfrentamento comunitário ao impacto do som automotivo}

Diante do impacto negativo referente a perturbação provocada pelos paredões, que segundo os moradores tocam de um dia ao outro, a comunidade vem se organizando para combater o problema. Ao longo dos anos, o grupo organizou abaixo-assinados, protocolaram ações na justiça, acionaram a imprensa, além de prestarem constantemente queixas policiais, que respondem com medidas paliativas. Essas ações repercutem tensões entre os moradores, proprietários dos carros e dos bares, que se sentem ameaçados com a possível ausência do atrativo musical.

Preocupados com o aumento dos paredões na orla em decorrência das festividades no final do ano de 2017, os moradores decidiram organizar ações prévias de conscientização da comunidade, em parceria com pesquisadores, profissionais da saúde, estudantes e militantes do movimento social, para que alguma medida fosse tomada quanto a perturbação prevista. O grupo visitou os moradores do centro histórico, um ambulatório de saúde e a Unidade Básica de Saúde (UBS) da região impactada, bem como os representantes da guarda municipal, do Batalhão da Polícia Militar e o vice-prefeito.

Após este movimento a prefeitura tomou a medida de interditar uma via da orla todos os dias entre $17 \mathrm{~h}$ e $21 \mathrm{~h}$, atendendo também a um pedido da comunidade de utilizar o espaço para a prática de lazer e esporte. Esta medida aumentou o número de frequentadores e reduziu o ruído, apesar de que após a reabertura das vias, os carros retornam com os paredões. Os serviços de segurança reforçaram a fiscalização durante o período de fechamento, mas os moradores se queixam ainda da negligência da polícia mediante as denúncias realizadas via telefone na madrugada.

Um aspecto observado durante as visitas nos serviços de saúde foi o relato dos profissionais sobre os pacientes idosos e crônicos que fazem a utilização de psicotrópicos para conseguirem dormir. Essa informação também foi reforçada pelos moradores que possuem doenças crônicas, pois sentem-se piores durante a exposição ao ruído. Os jovens relataram dificuldades de dormir em sono profundo, além de terem que assistir televisão no volume máximo ou com o auxilio de uma caixa de som (Martins, 2018).

Para compreender sobre os efeitos da exposição dos moradores aos sons automotivos, os pesquisadores, estudantes e profissionais da saúde envolvidos na ação decidiram investigar os documentos ou recomendações de organizações da saúde. As publicações OMS/Europa utilizam evidências dos efeitos do ruído na saúde para identificar as necessidades de grupos vulneráveis e fornecer orientações técnicas e políticas de proteção a saúde.

\section{Revisão das publicações da OMS/Europa.}

Os documentos foram analisados e resumidos os pontos que mais se relacionavam aos efeitos da exposição prolongada aos sons automotivos, sendo eles: efeitos biológicos, qualidade do sono, bem-estar e condições médicas. A Tabela 1 sintetiza as publicações, que indicam os prejuízos do ruído na saúde humana, atividades diárias (e.g. escola, trabalho, casa e nos momentos de lazer), no sono, efeitos cardiovasculares, psicofisiológicos, desempenho, nas respostas de aborrecimento e as mudanças no comportamento social.

O guia publicado em 2018, que atualiza as publicações anteriores sobre os riscos para a saúde decorrentes do ruído ambiental, recomenda aos Estados Membros da OMS/Europa esforços na prevenção e controle (Héroux et al., 2020). Duas novas fontes de ruído foram apresentadas: turbinas eólicas e ruído de lazer. O ruído do lazer, neste contexto, refere-se a todas as fontes de ruído que as pessoas estão expostas em atividades de lazer, tais como boates, pubs, aulas de fitness, eventos esportivos, concertos ou locais de música ao vivo e ouvir música alta através de aparelhos auditivos pessoais (e.g. paredão de som).

Os guias em geral orientam seguir as diretrizes e regulamentos legais e limitar os riscos de aumento do dano auditivo, tanto em crianças quanto em adultos. Recomenda-se fortemente medidas políticas para evitar a exposição acima dos valores de diretriz para ruído médio e exposições a ruído de evento único e de impulso. Isto é particularmente relevante uma vez que muitas pessoas podem ser expostas ao risco de perda auditiva através do uso de aparelhos auditivos pessoais. No entanto, não há evidências suficientes para recomendar um tipo de intervenção sobre esta fonte (WHO/Europe, 2018).

As estratégias metodológicas de evidências sobre os impactos do ruído na saúde recomendam dois protocolos: um para os efeitos na saúde e outro para as intervenções 
Tabela 1. Publicações da OMS/Europa sobre o ruído ambiental

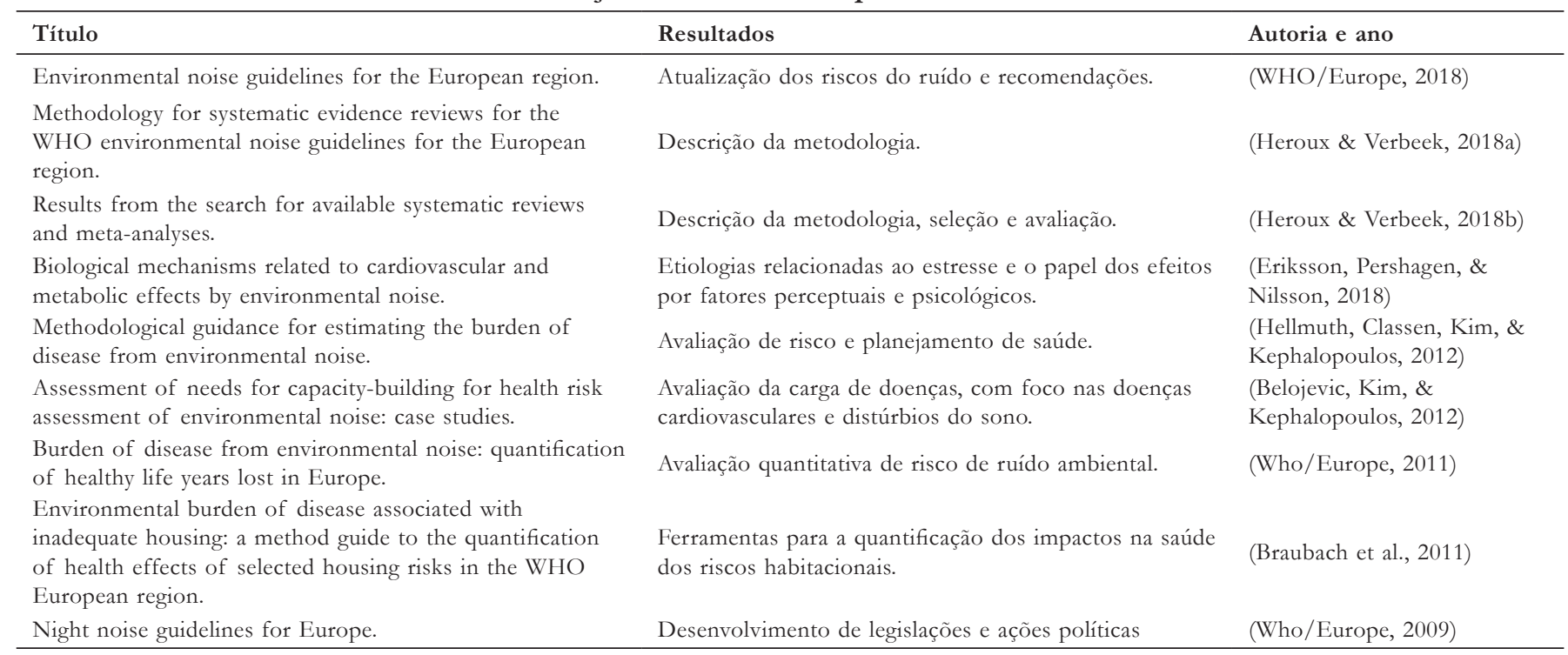

Fonte: Escritório Regional Europeu da OMS

(Heroux \& Verbeek, 2018a, 2018b). Os resultados da revisão sistemática foram: efeitos sobre o sono; incômodo; comprometimento cognitivo; qualidade de vida; saúde mental e bem-estar; doenças cardiovasculares e metabólicas; deficiência auditiva e zumbido; resultados adversos ao nascimento. O ruído ambiental foi definido como ruído emitido a partir de todas as fontes, exceto fontes de exposição ao ruído ocupacional nos locais de trabalho.

Os documentos apresentados na tabela 2 indicam que o ruído noturno provoca níveis altos de "efeito adverso não observado no corpo e na mente"(Who/Europe, 2009). Este é um conceito da Toxicologia, definido pela maior concentração não detectável nos exames de laboratório, mas que provoca alteração adversa na morfologia, capacidade funcional, crescimento, desenvolvimento ou tempo de vida do organismo. Em pouco tempo o nível alto e prolongado de exposição ao som torna as pessoas dependentes à ele, que associado ao consumo de álcool e outras drogas, pode gerar efeitos patológicos graves, como o infarto do miocárdio ou a aceleração das atividades fisiológicas.

Alguns grupos são mais vulneráveis ao barulho (WHO/Europe, 2018). Como as crianças passam mais tempo na cama do que os adultos, elas ficam mais expostas ao ruído noturno. Os doentes crônicos e idosos são os mais vulneráveis aos distúrbios emocionais provocados pela excessiva irritação. Isso também ocorre com as mulheres grávidas e os pacientes terminais. Os trabalhadores que cumprem horário comercial são os que estão em maior risco, porque a estrutura do sono está sob estresse, o que pode ser fator para doenças no sistemas nervoso, cardíaco, urinário e respiratório. Além disso, os mais pobres, que não podem subsidiar moradias em áreas residenciais tranquilas ou possuir domicílios com isolamento acústico adequado provavelmente sofrem desproporcionalmente (Braubach, Jacobs, \& Ormandy, 2011).

\section{Tabela 2. Sumário dos efeitos da exposição prolongada ao barulho noturno}

\begin{tabular}{ll}
\hline \multirow{2}{*}{ Efeitos biológicos } & Mudanças na atividade cardiovascular \\
& Mudanças na duração, estrutura e fragmentação do sono \\
& Despertar durante a noite e/ou muito cedo pela manhã \\
Qualidade do sono & Prolongamento do início do período ou dificuldade em pegar no sono \\
& Fragmentação e redução do tempo de sono \\
Bem-estar & Motilidade média aumentada quando dorme \\
Condições médicas & Distúrbio de sono auto relatados \\
\hline
\end{tabular}

Fonte: Escritório Regional Europeu da OMS 


\section{Conclusão}

O objetivo deste trabalho foi relatar as ações comunitárias de um grupo de moradores e parceiros impactados por um empreendimento hidrelétrico. Diante das diversas ações tomadas e dos avanços alcançados, os paredões de som continuam a importunar as pessoas que vivem próximo aos locais dos bares. Nota-se que o barulho de lazer está introjetado no cotidiano das pessoas. O som que se escuta na rua afeta a rotina e com o tempo começa-se a fazer tudo no volume alto, diminuindo a habilidade da comunicação.

Os relatórios da OMS/Europa comprovam que é impossível sobreviver sob tanto barulho. Sofrem os humanos, outros animais (domésticos e selvagens) e a estrutura do patrimônio histórico, que não suportam a vibração. Infelizmente ainda é um problema pouco associado pelas pessoas quando vão consultar os profissionais de saúde sobre os agravos na saúde como diabetes, hipertensão, ansiedade, alteração de humor, depressão, gastrite, surdez,com prevalência em idosos e crianças.

As informações deste relato são promissoras para uma área ainda em desenvolvimento no Brasil e que implica a articulação interdisciplinar em torno dos efeitos dos barulhos de lazer na saúde. O contexto em que a experiência ocorreu poderá auxiliar as futuras políticas públicas na mitigação de impactos provocados por projetos de larga escala e infraestrutura. A compreensão destes agravos por parte dos gestores, profissionais da saúde e da educação poderá auxiliar na mudança de atitude das pessoas e da comunidade quanto a prevenção.

\section{Referências}

Alves, L., Araújo, B., \& Pinto, Dé. (2014). Mapeamento acústico da concentração de denúncias de poluição sonora em Natal-RN. XV Encontro Nacional de Tecnologia Do Ambiente Construido, 1, 946-954. https://doi.org/10.17012/ entac2014.694

Belojevic, G., Kim, R., \& Kephalopoulos, S. (2012). Assessment of needs for capacity-building for health risk assessment of environmental noise: case studies. In European Commission Joint Research Centre. Retrieved from http://www.euro.who.int/en/health-topics/environment-and-health/ noise/publications

Brasil. (2018). Estatísticas do Registro Civil. Retrieved from https://biblioteca. ibge.gov.br/visualizacao/periodicos/135/rc_2017_v44_informativo.pdf

Braubach, M., Jacobs, D. E., \& Ormandy, D. (2011). Environmental burden of disease associated with inadequate housing: a method guide to the quantification of health effects of selected housing risks in the WHO European Region. In Summary report. Retrieved from http://www.euro.who. int/_data/assets/pdf_file/0003/142077/e95004.pdf

Bressane, A., Mochizuki, P. S., Caram, R. M., \& Roveda, J. A. F. (2016). A system for evaluating the impact of noise pollution on the population's health. Cadernos de Saude Publica, 32(5), 1-11. https://doi.org/10.1590/0102$-311 \mathrm{X} 00021215$

Carvalho e Silva, J. (2018). Impactos individuais e contextuais do deslocamento forçado na comunidade. Universidade de Brasília.

Carvalho e Silva, J., \& Ertzogue, M. H. (2015). Cosmologia, paisagem, lugar e o método fenomenológico : possíveis reflexões em uma cidade impactada por barragem. PRACS: Revista de Humaninadades Do Curso de Ciências Sociais Da UNIFAP, 8(1), 11-24. Retrieved from https://periodicos.unifap.br/ index.php/pracs/article/view/1149

CELTINS. (1996). RIMA Relatório de Impacto sobre o Meio Ambiente. Palmas.

Dos Santos, N. F., \& Rodrigues, F. D. J. (2019). Paredão na calçada, polícia na porta: conflitos vicinais e transformação da diversão nas periferias de São Miguel dos Campos - Alagoas. Mediações - Revista de Ciências Sociais, 24(2), 278. https://doi.org/10.5433/2176-6665.2019v24n2p278

Eriksson, C., Pershagen, G., \& Nilsson, M. (2018). Biological mechanisms related to cardiovascular and metabolic effects by environmental noise. In European Commission Joint Research Centre. Retrieved from http://www. euro.who.int/en/health-topics/environment-and-health/noise/publications

Foschiera, A. A. (2009). Da barranca do rio para a periferia dos centros urbanos: a trajetória do Movimento dos Atingidos por Barragens face às politicas do setor elétrico no Brasil. Universidade Estadual Paulista.

Hebenbrock, J. M. S. (2012). Calcinha Preta, Garota Safada e Aviões do Forró: a Cultura do Paredão Eletrônico no sertão cearense. XXXV Congresso Brasileiro de Ciências Da Comunicaşão, 1(1), 1-14. Retrieved from http://www. intercom.org.br/papers/nacionais/2012/resumos/R7-0370-1.pdf

Hellmuth, T., Classen, T., Kim, R., \& Kephalopoulos, S. (2012). Methodological guidance for estimating the burden of disease from environmental noise. In European Commission Joint Research Centre. Retrieved from http://www. euro.who.int/en/health-topics/environment-and-health/noise/publications

Heroux, M.-E., \& Verbeek, J. (2018a). Methodology for systematic evidence reviews for the WHO environmental noise guidelines for the European region. In European Commission Joint Research Centre. Retrieved from http:// www.euro.who.int/en/health-topics/environment-and-health/noise/ publications

Heroux, M.-E., \& Verbeek, J. (2018b). Results from the search for available systematic reviews and meta-analyses. In European Commission Joint Research Centre. Retrieved from http://www.euro.who.int/en/health-topics/ environment-and-health/noise/publications

Héroux, M. E., Babisch, W., Belojevic, G., Brink, M., Janssen, S., Lercher, P., ... Verbeek, J. (2020). WHO environmental noise guidelines for the European Region. Euronoise 2015, 2589-2593.

Jesus, B. M. De, \& Ertzogue, M. H. (2018). Sobre a saudade de um rio: perdas simbólicas dos ribeirinhos do Tocantins. DESAFIOS - Revista Interdisciplinar Da Universidade Federal Do Tocantins, 5(Especial), 169-177. https://doi. org/10.20873/uft.2359-3652.2018v5nEspecialp169

Martins, L. F. R. (2018). Vida urbana: poluição sonora na Orla Beira Rio. Jornal Do Tocantins, pp. 8-9. Retrieved from https://www.jornaldotocantins.com. br/editorias/estado/poluição-sonora-na-orla-beira-rio-1.1583246

Messias, N. C. (2012). Porto Nacional: patrimônio cultural e memória. Goiânia: Editora da PUC Goiás.

Oliveira, M. F. (1997). Um Porto no sertão: cultura e cotidiano em Porto Nacional 1880/1910. Universidade Federal de Goiás.

Parente, T. G. (2015). (In)visibilidade de atores no processo de reassentamentos da Usina Hidrelétrica Luís Eduardo Magalhães, no Tocantins. Revista Territórios e Fronteiras, 8(1), 149. https://doi.org/10.22228/rt-f.v8i1.296

Who/Europe. (2009). Night noise guidelines for Europe. In European Commission Joint Research Centre. Retrieved from http://www.euro.who.int/en/ health-topics/environment-and-health/noise/publications

Who/Europe. (2011). Burden of disease from environmental noise: quantification of healthy life years lost in Europe. In European Commission Joint Research Centre. Retrieved from http://www.euro.who.int/en/health-topics/ environment-and-health/noise/publications

WHO/Europe. (2018). WHO environmental noise guidelines for the European Region. European Commission Joint Research Centre, 181. Retrieved from http://www.euro.who.int/en/health-topics/environment-and-health/ noise/publications

Zitzke, V. A. (2007). A Rede Sociotecnica da Usina Hidrelétrica do Lajeado (To) e os Reassentamentos Rurais das famílias atingidas. Universidade Federal de Santa Catarina.

Submetido em: 4-6-2020

Aceito em: $21-7-2020$ 